\title{
Backward Wave Cyclotron-Maser Emission in the Auroral Magnetosphere
}

\author{
D. C. Speirs, ${ }^{1, *}$ R. Bingham, ${ }^{1,2}$ R. A. Cairns, ${ }^{3}$ I. Vorgul, ${ }^{3}$ B. J. Kellett, ${ }^{2}$ A. D. R. Phelps, ${ }^{1}$ and K. Ronald ${ }^{1}$ \\ ${ }^{1}$ Department of Physics, SUPA, University of Strathclyde, Glasgow, Scotland G4 ONG, United Kingdom \\ ${ }^{2}$ Rutherford Appleton Laboratory, Chilton, Didcot, Oxon, England OX11 0QX, United Kingdom \\ ${ }^{3}$ School of Mathematics and Statistics, University of St. Andrews, Fife, Scotland KY16 9SS, United Kingdom
}

(Received 25 October 2013; published 7 October 2014)

\begin{abstract}
In this Letter, we present theory and particle-in-cell simulations describing cyclotron radio emission from Earth's auroral region and similar phenomena in other astrophysical environments. In particular, we find that the radiation, generated by a down-going electron horseshoe distribution is due to a backwardwave cyclotron-maser emission process. The backward wave nature of the radiation contributes to upward refraction of the radiation that is also enhanced by a density inhomogeneity. We also show that the radiation is preferentially amplified along the auroral oval rather than transversely. The results are in agreement with recent Cluster observations.
\end{abstract}

DOI: 10.1103/PhysRevLett.113.155002

The electron cyclotron-maser instability plays a significant role in the generation of highly nonthermal, polarized radio emission from the auroral region of planets and stars having a dipolelike magnetic field [1,2]. More recently, this radiation model has been extended to a variety of astrophysical environments including Blazar jets, collisionless shocks, and brown dwarfs [3-7]. Planetary auroral radio emission has been a topic of interest for many years with several mechanisms having been proposed [8-15]. As the dispersion relation describing the electromagnetic wave growth only depends on the factor by which the magnetic field increases and on the ratio of plasma and cyclotron frequencies, the mechanism can scale effectively over many orders of magnitude both in wavelength and spatial scale. Blazar jets, in particular, represent a prime example of this scalability [3]. Generated perpendicular to the accretion disk of super massive black holes, they can extend over many thousands of light years and have been observed to generate highly nonthermal radio emission at frequencies in the hundreds of $\mathrm{GHz}$ range. It has been suggested that small scale magnetic mirrors or convergent flux tubes may be formed within the jet via hydrodynamic instabilities or shocks, providing the means of generating the required electron velocity distribution Bing2003, Begel2005. In Earth's auroral region, observations by the Viking spacecraft $[11,16]$ and the Fast Auroral Snapshot (FAST) satellite [14] led to the suggestion that the free energy source for AKR is a population of downward accelerated electrons having a large perpendicular velocity produced by a combination of parallel accelerating electric fields and converging magnetic field lines. The electron distribution takes the form of a horseshoe or crescent shape due to the first adiabatic invariance as the electrons move into the stronger magnetic field. The FAST satellite $[13,14,17]$ clearly demonstrates a strong correlation between the electromagnetic wave emission and the occurrence of the horseshoe distribution [13].
PACS numbers: 94.05.Dd, 94.30.Aa, 96.12.Wx, 98.62.Nx

Further observations by FAST of localized double-layer structures within the auroral density cavity have combined our understanding of auroral particle acceleration with the auroral kilometric radiation (AKR) emission process [18]. The associated formation of drifting electron and ion holes has also been presented as an explanation for striated AKR, with models of cyclotron-maser emission occurring discretely within electron holes [19] and explanations of coupling dynamics, spectral content, and escape of the radiation from the electron-hole structures [20]. In all cases, the cyclotron-maser instability is presumed to be driven by an electron horseshoe or modified horseshoe-ring distribution. Scaled laboratory experiments have demonstrated the potential for such horseshoe distributions to generate narrowband cyclotron-maser emission at $\mathrm{GHz}$ frequencies over distances of a few meters [21-25].

Although the generation mechanism is well established, there remain important questions with regard to how the radiation may propagate within the parent auroral density cavity and ultimately escape [26]. Cairns et al. [27] have demonstrated how the radiation, generated just below the electron cyclotron frequency, may successfully couple onto the upper branch of the plasma dispersion relation and escape. This model assumes near field-aligned $R$ mode polarization prior to escape, as observed by the Cluster satellite mission [28]. However, it is well known that the radiation is generated near perpendicular to the magnetic field in the $X$ mode [17] and will undergo strong resonant absorption when the radiation encounters the second harmonic layer [26]. The absorption is maximum when the radiation propagates perpendicular to the magnetic field and goes to zero as the radiation propagates parallel to the magnetic field [10]. The manner by which the radiation may effectively be redirected along the magnetic field and ultimately escape is a question recently addressed by Mutel [28] and Meniett [29] with a model of upward refraction 


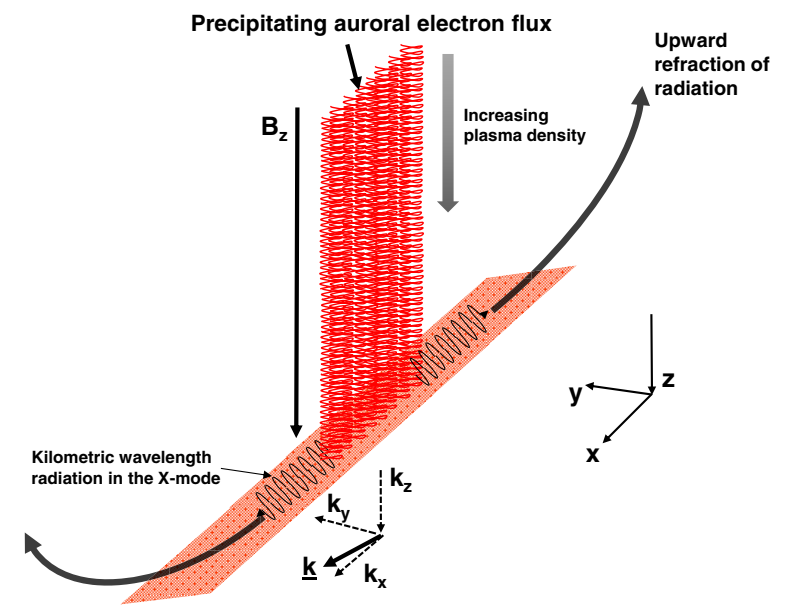

FIG. 1 (color online). Diagrammatic representation of cyclotron emission in the $X$ mode, with upward refraction of the generated radiation tangential to the auroral oval.

that is due to altitude variation in the background plasma density and an initial radiation propagation vector that is mildly against the direction of the electron flux (negative axial wave number), see Fig. 1. They also concluded that there was enhanced growth of the radiation tangential to the auroral oval [29]. Current theoretical models for $X$ mode generation via the cyclotron-maser instability do not predict growth with a negative axial wave number; however, we have developed a new theoretical model that clearly demonstrates an enhanced growth rate for finite negative axial wave numbers and a moderate electron energy spread within a horseshoe shaped electron velocity distribution $[11,13]$. This model is verified by the results of full 3D particle-incell (PIC) code simulations, demonstrating, for the first time, that auroral cyclotron-maser emission is, indeed, characterized by a finite negative axial wave number.

The theoretical model assumes an analytic form for the electron distribution function with different pitch angles, energy spread and density ratios between the hot component making up the horseshoe element and a background Maxwellian component. This is then used in a dispersion relation for the $R X$ mode which is obtainable from the susceptibility tensor given by Stix [30]. We assume that the frequency is close to the electron cyclotron frequency, and that the Larmor radius is much less than the wavelength for typical electron velocities. This latter condition means that we need only consider the susceptibility to lowest order in $k_{\perp} v_{\perp} / \omega_{c e}$ where $\omega_{c e}=e B / \gamma m_{e}$ is the relativistic electron cyclotron frequency with $e$ the electron charge, $B$ the magnetic flux density, $\gamma$ the Lorenz factor and $m_{e}$ the electron rest mass. If we neglect all but the zero order terms, we get the cold plasma result. For a first approximation, we need only take account of the velocity distribution of the electrons in the resonant integral which involves $1 /\left(\omega-\omega_{c e}\right)$. In terms of momentum $p$ in the weak relativistic limit, we have $\omega_{c e} \approx \omega_{c e 0}\left(1-p^{2} / 2 m_{e}^{2} c^{2}\right)$, where $\omega_{c e 0}$ is the nonrelativistic electron cyclotron frequency. For the real part of the resonant integral, we can simply take the cold plasma value. Although this goes as $1 /\left(\omega-\omega_{c e 0}\right)$ and appears to be near singular at the resonance, the $1 /\left(\omega-\omega_{c e 0}\right)$ factors in the real part of the dispersion relation cancel out, and it behaves quite smoothly in the vicinity of the cyclotron frequency. It is not crucial to include small corrections to the cyclotron frequency in the real part of the dispersion relation. The refractive index $n$ for the $R X$ mode is given by $n^{2}=\epsilon_{\perp}-\epsilon_{x y}^{2} / \epsilon_{\perp}$, where $\epsilon_{\perp}$ and $\epsilon_{x y}$ are, respectively, the perpendicular and $x$ and $y$ dielectric tensor elements [30]. In the limit where the Larmor radius is small compared to the wavelength, the imaginary parts of all components of the dielectric tensor for near perpendicular propagation are approximately proportional to $\operatorname{Im}(D)=$ $\int d v_{\|} v_{\perp} d f / d v_{\perp}$ with $v_{\perp}$ determined as a function of $v_{\|}$ by the resonance condition $\omega-k_{\|} v_{\|}=\omega_{c e}$. This assumes that the relativistic factor $\gamma$ can be set equal to one everywhere except in the resonance condition. If we take a typical horseshoe distribution, produced from an initial Maxwellian with drift speed $0.25 \mathrm{c}$ and thermal velocity $0.015 \mathrm{c}$ subject to magnetic compression by a factor of 15 , the maximum growth occurs when the resonance curve passes through a fairly restricted area in velocity space, for which $\partial f / \partial v_{\perp}$ is maximized. The shape of the resonance curve is presented in Fig. 2(a), plotted for $\omega=0.97 \omega_{c e}$ and three different values of $n_{\|}=k_{\|} c / \omega$. The key point to note is that, for positive $n_{\|}$, the curve lies above the $n_{\|}=0$ curve, while for negative $n_{\|}$, it lies below. The other relevant point is that an increase in frequency moves the whole curve downwards, starting at a lower $v_{\perp}$ for $v_{\|}=0$. Combining these two pieces of information, it can be seen that, in order for the resonance curve to pass through the
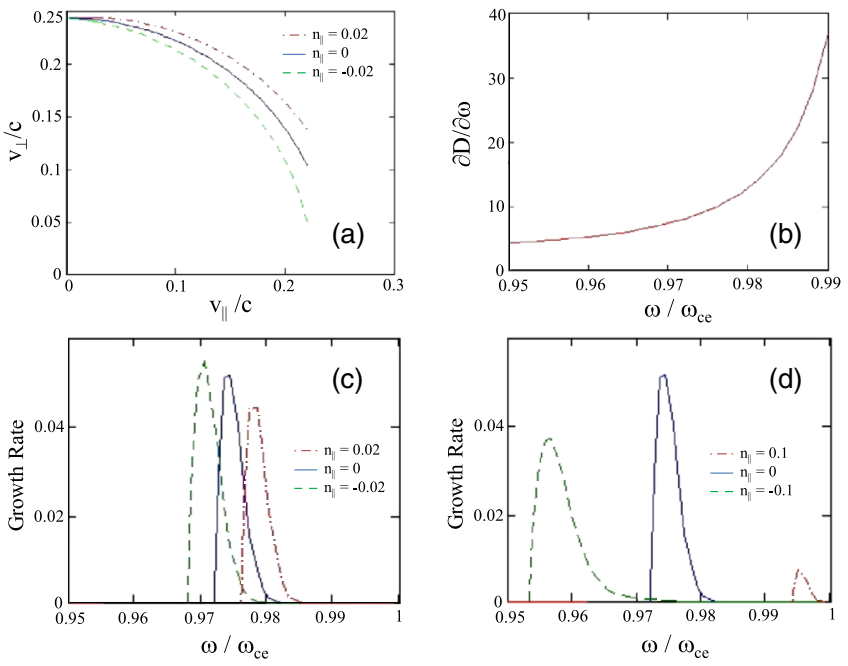

FIG. 2 (color online). (a) Resonance curves for $n_{\|}=0.02,0$, and -0.02 . (b) Gradient with respect to frequency of the dispersion function. (c) Relative growth rates for $n_{\|}=0.02,0$, and -0.02 . (d) Relative growth rates for $n_{\|}=0.1,0$, and -0.1 . 
region of maximum $\partial f / \partial v_{\perp}$, it must have a higher frequency when $n_{\|}$is positive than when $n_{\|}$is zero, so that the lower starting $v_{\perp}$ at $v_{\|}=0$ compensates for the smaller downwards curvature. Conversely, for negative $n_{\|}$, maximum growth is to be expected at a lower frequency.

When the frequency is chosen so that the imaginary part of the dielectric tensor is a maximum for each value of $n_{\|}$, then the imaginary part does not vary greatly for small values of $n_{\|}$, positive or negative. However, the growth rate is approximately given by $\operatorname{Im}(\omega)=(\operatorname{Im}(D)) /((\partial / \partial \omega)[\operatorname{Re}(D)])$, where $D=0$ is the dispersion relation. As a simple approximation to $D$, we just take the cold plasma result at perpendicular incidence, a reasonable approximation if $n_{\|}$is small and the Larmor radius is small compared to the perpendicular wavelength. The value of $\partial D / \partial \omega$ as a function of frequency is shown in Fig. 2(b), for $\omega_{p}=0.1 \omega_{c e}$. The crucial point to note is that, because of the proximity to the upper hybrid resonance, where $D$ has a singularity, the gradient shows a strong increase with frequency [Fig. 2(b)]. This means that the growth rate for positive $n_{\|}$components, where $\operatorname{Im}(D)$ is greatest at higher frequencies, is suppressed, while the net growth rate for lower $n_{\|}$components is enhanced. This is illustrated in Figs. 2(c) and 2(d), which show the relative growth rates for different values of $n_{\|}$.

If we go to larger values of $\pm n_{\|}$, the value of (1) tends to decrease since the resonance curve starts to pass through the region of optimum perpendicular gradient at a more oblique angle. This is illustrated in Fig. 2(d) where the oblique components now have $n_{\|}= \pm 0.1$. This demonstrates that the peak growth rate is for radiation at a few degrees from perpendicular in the opposite direction to beam propagation and the results are rather insensitive to the value of $\omega_{p}$ in the range $\omega_{p} \leq \omega_{c e}$. Beam spreading in velocity space tends to produce a reduced growth rate for negative $n_{\|}$over a smaller range of angles.

We compare the theoretical analysis with the results of PIC simulations, undertaken to study the evolution of the instability. The numerical model was defined using the particle-in-cell code VORPAL [31]. The simulation geometry comprised a Cartesian gridded region with an axial length of $144 \lambda_{c e}$ (where $\lambda_{c e}$ is the vacuum wavelength of radiation at the peak electron cyclotron frequency within the simulation), symmetric transverse dimensions of $5.4 \lambda_{c e}$ and perfectly matched layer boundaries in $x$ and $y$ to mitigate reflection and the formation of boundary resonant eigenmodes. A $20 \mathrm{keV}$ electron beam was injected parallel to an axial magnetic field increasing by a factor of 15 over $45 \lambda_{c e}$. The peak magnetic flux density was plateaued over the remaining $99 \lambda_{c e}$ of the beam propagation path to maintain cyclotron resonance for a significant number of Larmor steps. We considered two injected Gaussian electron velocity distributions of $2 \%$ and $6 \%$ energy spreads and a ratio of electron cyclotron frequency to plasma frequency of 20.
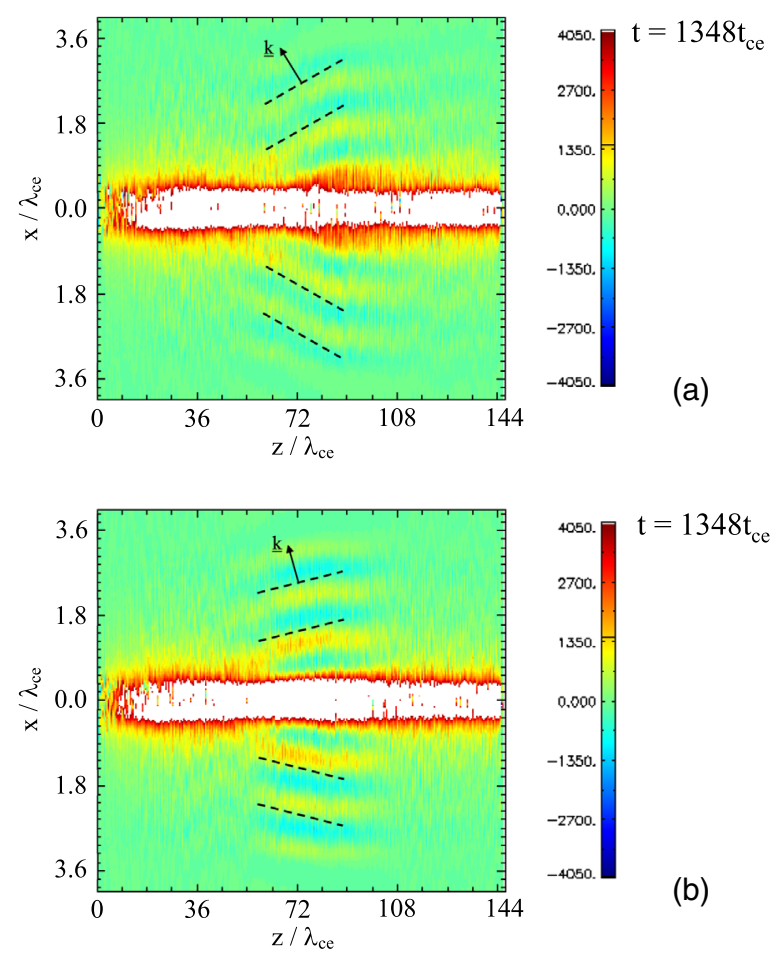

FIG. 3 (color online). Contour plot of $E_{y}$ in an $x-z$ plane at $t / t_{c e}=1348$ for (a) $2 \%$ and (b) $6 \%$ Gaussian energy spreads.

An analysis of the transverse electric field profile $E_{y}$ in an $x-z$ plane (Fig. 3) for 2\% and 6\% energy spreads reveals a well-defined and temporally modulated electromagnetic emission at an axial coordinate of $80 \lambda_{c e}$ polarized in the $X$ mode with a significant negative axial wave number (backward-wave component). The backward wave character of emission is evidenced by an oblique wave front propagation angle with respect to the axis of the system. Factoring in the differing axial scales in $x$ and $z$, the effective backward-wave angle for the $2 \%$ energy spread is $\sim 3^{\circ}$ from perpendicular - consistent with requirements for the aforementioned model of upward refraction of the generated radiation [29].

The analytical theory predicts that an increase in energy spread will have a marked effect on the wave vector of emission. It is evident in Fig. 3 that as the energy spread increases, there is, indeed, such a well-defined change in the wave vector. For the $6 \%$ energy spread, there is still a significant negative axial wave number to the emission, but representing a reduction in backward propagation angle to $\sim 2^{\circ}$ from perpendicular. This emission profile is not only indicative of a reduction in backward wave coupling with an increased energy spread, but a distinct curvature can also be observed in the wave front for the 6\% energy spread between $z=56 \lambda_{c e}$ and $84 \lambda_{c e}$. This curvature indicates a progressive shift towards perpendicular propagation with an increasing axial coordinate, showing the transverse electron velocity spread induced via energy transfer to the wave results in the peak growth occurring for decreasing 

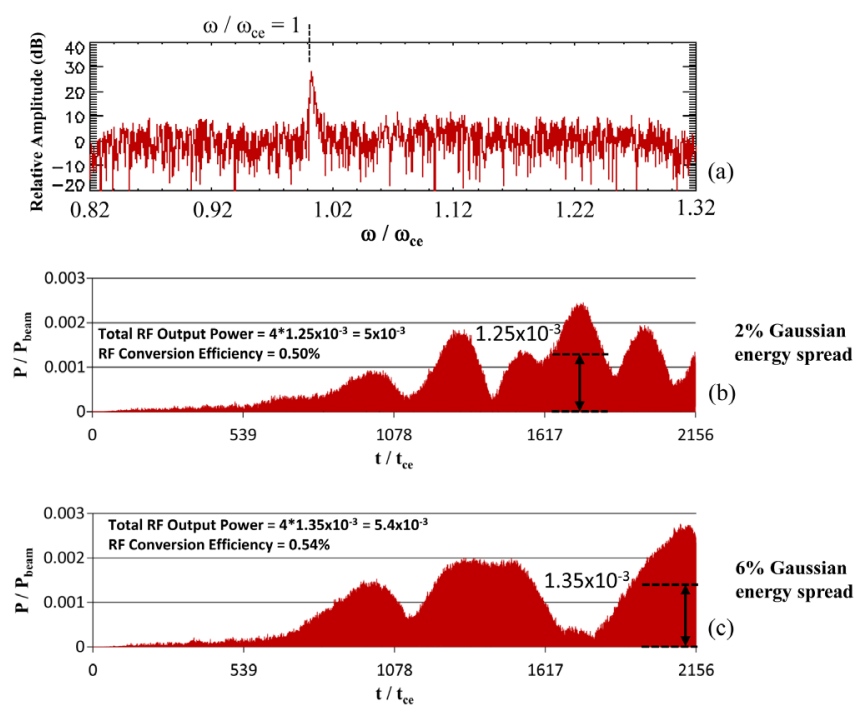

FIG. 4 (color online). (a) Fourier transform of $E_{y}$ total history at $t / t_{c e}=2156$ and $z=86.4 \lambda_{c e}$. (b) Radial Poynting flux across a $y-z$ plane over the length of the simulation for $2 \%$ and (c) $6 \%$ Gaussian electron energy spread.

axial wave numbers. This result is consistent with predictions of the analytical theory and has important implications for the dynamics of the horseshoe driven cyclotron-maser process.

Figure 4(a) contains a Fourier transform of $E_{y}$ at time $2156 t_{c e}$ and an axial position of $86 \lambda_{c e}$. A single spectral component is present at $\omega / \omega_{c e}=1$, corresponding to narrowband emission at the relativistic electron cyclotron frequency. Looking at the corresponding transverse Poynting flux over a $y-z$ plane in Fig. 4(b), a temporal modulation to the emission is evident, with an $810 t_{c e}$ lead time for significant growth of the radio frequency (rf) Poynting flux. A peak saturated output power of $P / P_{\text {beam }}=1.25 \times 10^{-3}$ is apparent (as the space-time average is $1 / 2$ of the plotted peak amplitude), which integrated over the transverse dimensions of the system (factoring in the four enclosing Poynting flux planes) equates to an $\mathrm{rf}$ conversion efficiency of $0.50 \%$.

A comparative look at the transverse Poynting flux for the 6\% energy spread in Fig. 4(c) reveals a significant change in temporal character, with the $2 \%$ energy spread exhibiting a highly modulated profile while the $6 \%$ energy spread yields a more stable output, with longer period temporal fluctuations and a marginally increased peak beam-wave conversion efficiency of $0.54 \%$. In both cases, however, the mean output power remains relatively unchanged. Therefore, it would appear that as the electron energy spread is increased, not only does the wave vector of emission tend from backward wave to near perpendicular, but the increased energy spread also leads to a more stable cyclotron-maser emission process.

Although the simulations previously outlined demonstrate the variation in emission topography as a function
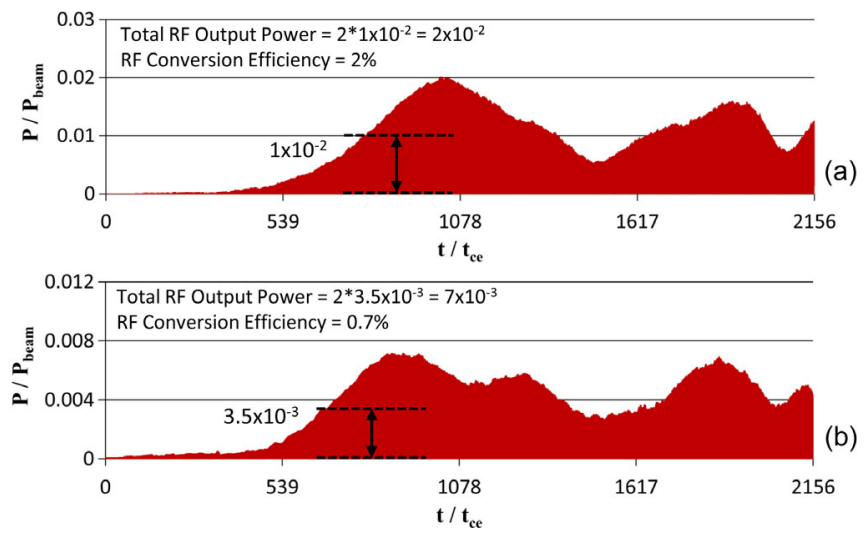

FIG. 5 (color online). (a) Radial Poynting flux across the $y-z$ plane. (b) Radial Poynting flux across the $x-z$ plane.

of energy spread, they do not account for growth of the emission over a large number of wavelengths. This effect has significant implications for the auroral magnetospheric case, where propagation tangential to the auroral cavity boundary is considered to increase the effective path length for upward refraction [28]. A 3D PIC simulation was used to study the evolution of the horseshoe-maser instability in a sheet electron beam, with a cross section of $9 \lambda_{c e} \times 0.6 \lambda_{c e}$. The parameters for this simulation are consistent with those of the previous cylindrical beam simulations, although the larger beam cross section yielded a reduction in electron number density and an increased ratio of electron cyclotron frequency to plasma frequency $\omega_{c e} / \omega_{p e}=120$. A horseshoe distribution was also defined with the $6 \%$ Gaussian energy spread and a continuous, static axial magnetic field to maintain the injected sheet aspect ratio of the electron beam over the simulation length.

The observed spectral output matches that of the initial cylindrical beam simulations, with a singular spectral component at $\omega / \omega_{c e}=1$. A contrast is encountered, however, in the transverse Poynting fluxes, presented over $y-z$ and $x-z$ planes, respectively, in Fig. 5. It is clear that across the larger transverse dimension of the sheet electron beam $(y-z$ plane $)$ there is a significant enhancement in output power, with a peak output of $P / P_{\text {beam }}=1 \times 10^{-2}$ compared with $3.5 \times 10^{-3}$ for emission across the narrower beam dimension $(x-z$ plane). The increased output power along the larger dimension of the sheet has significant implications, supporting the hypothesis of enhanced radiated power over an extended path tangential to the auroral plasma cavity boundary [28]. The net beam-wave conversion efficiency in this case is also significantly enhanced, amounting to $2.7 \%$ of the electron beam power. This demonstrates the significance of increased growth in radiation power over greater distances.

In conclusion, we have shown that the electron cyclotronmaser instability driven by a horseshoe-shaped velocity distribution has an inherently enhanced growth rate for wave propagation with finite negative $k_{\|}$corresponding to 
a backward-wave propagation angle a few degrees from perpendicular. This natural backward-wave character to the emission serves as a suitable precursor to the recently proposed model of upward refraction and field-aligned escape of terrestrial auroral kilometric radiation [28,29]. This also limits the potential for harmonic absorption at perpendicular propagation [26]. We have also demonstrated that the wave power increases over larger path lengths. This has bearing on the currently proposed model of AKR upward refraction, where the largest path length for refraction (tangential to the auroral oval) also corresponds to the path of most efficient power output [29].

This work was supported by EPSRC Grant No. EP/ G04239X/1. R. B. and K. R. would like to acknowledge fruitful discussions with D. Melrose.

*david.c.speirs@strath.ac.uk

[1] P. Zarka, Adv. Space Res. 12, 99 (1992).

[2] R. Bingham, R. A. Cairns, and B. J. Kellett, Astron. Astrophys. 370, 1000 (2001).

[3] M. C. Begelman, R. E. Ergun, and M. J. Rees, Astrophys. J. 625, 51 (2005).

[4] R. Bingham, D. C. Speirs, B. J. Kellett, I. Vorgul, S. L. McConville, R. A. Cairns, A. W. Cross, A. D. R. Phelps, and K. Ronald, Space Sci. Rev. 178, 695 (2013).

[5] R. A. Treumann, Astron. Astrophys. Rev. 13, 229 (2006).

[6] R. Bingham, B. J. Kellett, R. A. Cairns, J. Tonge, and J. T. Mendonca, Astrophys. J. 595, 279 (2003).

[7] G. Hallinan, A. Antonova, J. G. Doyle, S. Bourke, C. Lane, and A. Golden, Astrophys. J. 684, 644 (2008).

[8] C. S. Wu and L. C. Lee, Astrophys. J. 230, 621 (1979).

[9] R. M. Winglee and P. L. Pritchett, J. Geophys. Res. 91, 13531 (1986)

[10] D. B. Melrose, Instabilities in Space and Laboratory Plasmas (Cambridge University Press, Cambridge, England, 1986).

[11] P. Louarn, A. Roux, H. de Feraudy, D. Le Queau, and M. Andre, J. Geophys. Res. 95, 5983 (1990).

[12] P. Louarn and D. Le Queau, Planet. Space Sci. 44, 211 (1996).

[13] G. T. Delory, R. E. Ergun, C. W. Carlson, L. Muschietti, C. C. Chaston, W. Peria, J. P. McFadden, and R. Strangeway, Geophys. Res. Lett. 25, 2069 (1998).
[14] R. E. Ergun, C. W. Carlson, J. P. McFadden, G. T. Delory, R. J. Strangeway, and P. L. Pritchett, Astrophys. J. 538, 456 (2000).

[15] R. Bingham and R. A. Cairns, Phys. Plasmas 7, 3089 (2000).

[16] A. Roux, A. Hilgers, H. de Feraudy, D. Le Queau, P. Louarn, S. Perraut, A. Bahnsen, M. Jespersen, E. Ungstrup, and M. Andre, J. Geophys. Res. 98, 11657 (1993).

[17] P. L. Pritchett, R. J. Strangeway, R. E. Ergun, and C. W. Carlson, J. Geophys. Res. 107, 1437 (2002).

[18] R. Pottelette, M. Berthomier, and J. Pickett, Ann. Geophys. 32, 677 (2014).

[19] R. A. Treumann, W. Baumjohann, and R. Pottelette, Ann. Geophys. 29, 1885 (2011).

[20] R. A. Treumann, W. Baumjohann, and R. Pottelette, Ann. Geophys. 30, 119 (2012).

[21] D. C. Speirs, I. Vorgul, K. Ronald, R. Bingham, R. A. Cairns, A. D. R. Phelps, B. J. Kellett, A. W. Cross, C. G. Whyte, and C. Robertson, J. Plasma Phys. 71, 665 (2005).

[22] K. Ronald, D. C. Speirs, S. L. McConville, A. D. R. Phelps, C. W. Robertson, C. G. Whyte, W. He, K. M. Gillespie, A. W. Cross, and R. Bingham, Phys. Plasmas 15, 056503 (2008).

[23] S. L. McConville, D. C. Speirs, K. Ronald, A. D. R. Phelps, A. W. Cross, R. Bingham, C. W. Robertson, C. G. Whyte, W. He, K. M. Gillespie, I. Vorgul, R. A. Cairns, and B. J. Kellett, Plasma Phys. Controlled Fusion 50, 074010 (2008).

[24] D. C. Speirs, S. L. McConville, K. M. Gillespie, K. Ronald, A. D. R. Phelps, A. W. Cross, R. Bingham, C. W. Robertson, C. G. Whyte, I. Vorgul, R. A. Cairns, and B. J. Kellett, Plasma Phys. Controlled Fusion 50, 074011 (2008).

[25] A. G. Shalashov, S. V. Golubev, E. D. Gospodchikov, D. A. Mansfeld, and M.E. Viktorov, Plasma Phys. Controlled Fusion 54, 085023 (2012).

[26] D. B. Melrose and G. A. Dulk, Astrophys. J. 259, 844 (1982).

[27] R. A. Cairns, I. Vorgul, and R. Bingham, Phys. Rev. Lett. 101, 215003 (2008).

[28] R. L. Mutel, I. W. Christopher, and J. S. Pickett, Geophys. Res. Lett. 35, L07104 (2008).

[29] J. D. Menietti, R. L. Mutel, I. W. Christopher, K. A. Hutchinson, and J. B. Sigwarth, J. Geophys. Res. 116, A12219 (2011).

[30] T. H. Stix, Waves in Plasma (AIP, New York, 1992).

[31] C. Nieter and J. R. Cary, J. Comput. Phys. 196, 448 (2004). 